\title{
Microarray expression profile analysis of aberrant long non-coding RNAs in esophageal squamous cell carcinoma
}

\author{
JUAN YAO ${ }^{1}$, JUN-XING HUANG ${ }^{1}$, MEI LIN ${ }^{2}$, ZHENG-DONG WU ${ }^{1}$, HONG YU ${ }^{3}$, PENG-CHENG WANG ${ }^{4}$, \\ $\mathrm{JUN} \mathrm{YE}^{2}$, PING CHEN ${ }^{3}$, JING WU ${ }^{5}$ and GUO-JUN ZHAO ${ }^{1}$ \\ Departments of ${ }^{1}$ Oncology, ${ }^{2}$ Experiment Center, ${ }^{3}$ Pathology and ${ }^{4}$ Thoracic Surgery, Taizhou People's Hospital \\ Affiliated to Nantong and Jiangsu University, Taizhou, Jiangsu 225300; ${ }^{5}$ Department of Oncology, \\ The People's Hospital of Deyang, Chengdu University of TCM, Deyang, Sichuan 618000, P.R. China
}

Received January 3, 2016; Accepted March 1, 2016

DOI: $10.3892 /$ ijo.2016.3457

\begin{abstract}
Increasing evidence indicates that long non-coding RNA (lncRNA) plays an important role in tumorigenesis. However, the function and regulatory mechanism of lncRNAs are still unclear in esophageal squamous cell carcinoma (ESCC). To address this challenge, we screened lncRNAs expression profiles in 3 pairs of ESCC and matched noncancerous tissues by microarray assay and identified the relationship between lncRNAs expression in ESCC tissue and clinicopathological characteristics and prognosis of patients with ESCC. We found 182 lncRNAs that were significantly differently expressed in ESCC tissues versus the matched non-cancerous tissues. Gene ontology and pathway analysis results suggested that the primary biological processes of these genes were involved in extracellular matrix, immune responses, cell differentiation and cell proliferation. Through cis and trans analyzing, we found 4 lncRNAs (ENST00000480669, NONHSAT104436, NONHSAT126998 and NONHSAT112918) may play important roles in tumorigenesis of ESCC. The four lncRNAs were checked in 73 patients with ESCC. The results showed that they mainly related to tumor metastasis. Kaplan-Meier survival analysis showed that high expression of NONHSAT104436, NONHSAT126998 and low expression of ENST00000480669 were related to poor 3 -year overall survival $(\mathrm{P}=0.003,0.032$ and 0.040 , respectively). Multivariate analysis showed that NONHSAT104436 was an independent prognostic factor $(\mathrm{P}=0.017)$. Thus we concluded that, lncRNAs showed differently expression patterns in ESCC versus matched noncancerous tissues, and aberrantly expressed lncRNA may play important roles in ESCC development and progression.
\end{abstract}

Correspondence to: Dr Jun-Xing Huang, Department of Oncology, Taizhou People's Hospital Affiliated to Nantong and Jiangsu University, 210 Yingchun Road, Taizhou, Jiangsu 225300, P.R. China E-mail: hjxtz@sina.cn

Key words: lncRNAs, esophageal squamous cell carcinoma, microarray analysis, ESCC clinicopathologic feature, ESCC prognosis
Interestingly, the overexpression of NONHSAT104436 was tightly correlated with distant metastasis and, poor survival rate, which might indicate that NONHSAT104436 might play a very important part in ESCC tumor progression.

\section{Introduction}

Esophageal cancer ranks as the sixth most common cancer death in the whole world (1). This disease is usually classified into EAC (esophagus adenous cancer) and ESCC (esophageal squamous cell carcinoma) based on histological types. EAC maily occurs in European and American countries, while ESCC has a high incidence of in China, accounting for $>90 \%$ of esophageal cancer. Despite decline in mortality over the past ten years, the prognosis of ESCC is still very poor and the mortality of esophageal carcinoma ranks the fourth cancer death in China (2). The challenge ahead is that tiology and pathogenesis of ESCC are not yet clearly understood. The incidence varies significantly among different regions (1). Recent studies have found that genetic abnormality is one of the major causes of ESCC indicating that the occurrence of ESCC may be related to the environmental factors, as well as genetic factors (3). Therefore, to achieve early accurate diagnosis, better curative effect and prognosis assessment of ESCC, we need to understand the pathogenesis at genomic level (4).

It is known that single stranded small molecule RNA-nonencoding RNAs (microRNAs), with a length of $21-25 \mathrm{nt}$ basic group, play a negative regulatory function in post transcriptional activity. A large number of studies have described the role of microRNA in tumorigenesis, development and metastasis of cancer (5-8). Additionally, non-encoding RNAs with long chain (long non-coding RNAs, lncRNAs) are a branch of non-encoding RNA transcript with $>200$ nucleotides in length and account for $80 \%$ of non-encoding RNA or more (9-11). LncRNAs mainly achieve the regulation of gene expression in three levels, which are epigenetic regulation, transcriptional regulation, and post-transcriptional regulation $(12,13)$. Considering the number, type, function and action mechanism of lncRNAs are far more abundant than miRNA, and lncRNAs may be the core of RNA world (14), increasing number of studies show that IncRNAs have a great potential to be served as biomarkers for tumorigenesis, metastasis and 
prognosis (15-17), and they are likely to be a new target for cancer therapy (18-20).

Therefore, we explored the potential roles of lncRNAs involved in ESCC in this study. We performed a genome wide profiling of lncRNA expression, and investigated the potential function of these distinguishable lncRNAs, and predicted lncRNAs target genes, and observed the relationship between expression level of lncRNAs and clinicopathological features, prognosis in patients with ESCC to find new bio-molecular markers.

\section{Materials and methods}

Patients and tissue samples. ESCC tissue samples and matched non-cancerous tissues $\geq 2 \mathrm{~cm}$ away from the edge of tumor tissues used in this research were from 76 ESCC patients who underwent surgical operation from March 2012 to October 2012 in Department of Thoracic Surgery, Taizhou People's Hospital Affiliated to Nantong and Jiangsu University. All patients signed written consent before esophagus resection. All specimens were stored at $-80^{\circ} \mathrm{C}$ within $10 \mathrm{~min}$ of the resection. ESCC was confirmed by pathology, and clinical data including age, sex, tumor size, $\mathrm{T}$ stage, $\mathrm{N}$ stage, $\mathrm{M}$ stage and TNM stage were available for all the cases selected. We extracted 3 tissues for microarray assay, while the other 73 tissues were examined by qRT-PCR for clinicopathologic analysis. The study was conducted in compliance with Institutional Ethics Committee of Taizhou People's Hospital Affiliated to Nantong and Jiangsu University and the Helsinki Declaration.

RNA extraction. RNA was extracted from 76 pairs of frozen ESCC tissues and matched adjacent non-cancerous tissues by TRIzol reagent kit (Invitrogen, CA, USA). The primary procedures were according to the manufacturer's protocol. The total RNA was subpackaged separately and preserved at $-80^{\circ} \mathrm{C}$. The concentration and purity of RNA was detected by UV spectrophotometer according to the absorbance values at 260 and $280 \mathrm{~nm}$ of wavelength.

Microarray analysis. Agilent Human lncRNA Microarray V2.0 (4*180K; Design ID, 062918; containing 46,506 lncRNAs) was used to analyze the lncRNA expression profiling of tumor tissues from ESCC patients. The lncRNA probes on gene chips were based on the well-known lncRNAs from Agilent_ncRNA, lncRNAdb, GencodeV13, $\mathrm{H}$-invDB, NONCODEV3, RefSeq, ultra-conserved region encoding lncRNAs (UCR), UCSC_lincRNAs Transcripts and Ensembl. Three ESCC tissues and three matched noncancerous tissues were analyzed by microarray as follows: i) 200-ng of total RNA from each specimen was applied to generate synthetic double stranded cDNA by Quick Amp Labeling kit, One-Color (Agilent p/n 5190-2305); ii) subsequently, the double stranded cDNA as a template was transcribed into cRNA by RNeasy Mini kit (Qiagen $\mathrm{p} / \mathrm{n}$ 74104) and labeled with Cy3-dCTP; iii) labeled cRNAs were hybridized to the gene microarray; iv) the microarrays were washed and scanned by an Agilent G2505C Microarray Scanner; v) the raw data were analyzed from array images by Feature Extraction software (version10.7.1.1, Agilent
Technologies). The standardized data analyses and subsequent data processing were done by Genespring (version 12.5, Agilent). The microarray hybridization was performed by Outdo Biotech, Shanghai, China.

Quantitative real-time polymerase chain reaction. In accordance to the manufacturer's protocol (Takara, Dalian, China), $2 \mu \mathrm{g}$ of the above total RNA extracted from ESCC tissues and matched non-cancerous tissues was reverse transcribed to cDNA, respectively. Additionally, then the real-time PCR reactions were executed by SYBR PrimeScript (Takara) and the ABI7900 (Applied Biosystems, CA, USA) as follows: i) initial denaturation for $30 \mathrm{sec}$ at $95^{\circ} \mathrm{C}$; ii) 40 cycles for $5 \mathrm{sec}$ at $95^{\circ} \mathrm{C}$ and for $30 \mathrm{sec}$ at $59^{\circ} \mathrm{C}$. Each sample was executed in triplicate. GAPDH was used as reference. The expression levels of lncRNAs were calculated by the $2^{-\Delta \Delta C T}$ method. The primer sequences are summarized in Table I.

LncRNA co-expression analysis. For each significant differentially expressed lncRNA, we calculate the Pearson correlation coefficients (PCCs) of its expression value with expression value of each mRNA. The absolute PCCs value $>0.8$ was considered meaningful. The PCCs value $\leq 0.8$ indicated negative correlation, and the value $>0.8$ indicated positive correlation. The P-value $<0.05$ was considered significant. DAVID (http://david.abcc.ncifcrf.gov/gene2gene. jsp) functional annotation database was used to analyze these correlative genes.

GO and KEGG analysis. The interrelated coding genes were reassigned to functional groups by Gene Ontology (GO: http:// www.geneontology.org) and Kyoto Encyclopedia of Genes and Genomes (KEGG: http://www.genome.jp/kegg) analysis. A brief overview of the process was as follows: firstly, we computed coexpressed mRNAs with each differentially expressed lncRNA, and then made up functional enrichment analysis for the set of coexpressed mRNAs. The enriched functional terms were used to predict functional term of appointed lncRNA. Ultimately, we applied hypergeometric cumulative distribution function to compute the enrichment of functional term in annotation of coexpressed mRNAs. The functional enrichment prediction of IncRNAs was based on biological processes, molecular function, cellular component and specific pathways.

Cis analysis. IncRNAs regulate the target gene expression by cis or trans mechanism. For analysis of the cis regulatory roles, the co-expressed IncRNAs-mRNAs were transcribed from the same local chromatin. Therefore, cis analysis could be an effective way to predict the target genes of lncRNAs. The potential cis-regulated mRNAs of lncRNAs had to meet the two following conditions: i) the mRNAs loci to be within $300 \mathrm{kbp}$ windows of the given lncRNA; ii) the PCCs of lncRNAs-mRNAs coexpression were statistically significant (PCCs $>0.8$ or $\mathrm{PCCs} \leq 0.8$; and $\mathrm{P}<0.05$ ). The cis-regulation regions were identified according to their location distributions by UCSC Genome Browser.

Trans analysis. Target genes of lncRNAs also can be determined by trans mechanism. Firstly, we worked out each 
Table I. The primer sequences used in RT-PCR.

\begin{tabular}{lll}
\hline Gene symbol & \multicolumn{1}{c}{ Forward primer } & \multicolumn{1}{c}{ Reverse primer } \\
\hline TCONS_00017817 & ACTCTCTGGGAGTTGAGAT & TAGGAATTGGATGACTCACGA \\
NONHSAT142035 & ATTTAAGACAAGTCTGGAAAGT & ATGGAAATAAGTTCTTAGAGTT \\
ENST00000480669 & CAGGCGCGGAGAGGCGCT & CTGCTCTGCTCACAGAAAC \\
NR_036468.1 & GCTTGGTGGTACATGAAGT & TGATGGACCAAATGGCTCTGA \\
XR_241594.1 & TGTTGCTGCTTTGCATTT & TGTGAGTTCTCACAGCAC \\
NONHSAT104436 & GTCATCTGCCCTTCTGTC & ACTGGCAAAGTCAGTAGAAT \\
ENST00000539535 & ACCAAGTCTTTCTTCCCATC & AGCAGTCTATGTCCAAAGTT \\
NONHSAT066293 & AAATCCTGGAACTGCTGAA & CAGGGCTTGGAATGTGAG \\
NONHSAT147911 & CGCTGATCCAGTGACAAT & TTGTGGTTGGAGGAGCTT \\
XR_248864.1 & GGAGTTATTAGGGTGCATCC & TCTAGCTTAGAAGTCCTCGG \\
NONHSAT112918 & GGTCCTACAGGGACTTGA & ATTTCCTTATGTTGCTGCCA \\
NONHSAT126998 & ATGACCAAACAAGGGTTAGT & CATAGGTCAAGAGTGAGGAT \\
GAPDH & GAGTCAACGGATTTGGTGGT & TTGATTTTGGAGGGATCTCG
\end{tabular}

differentially expressed IncRNA co-expressing coding genes and transcription factor in ENCODE (Encyclopedia of DNA Elements) (21), then we calculated the significance of differential genes enrichment in each TF term via hypergeometric distribution test method. A P-value enriched with significance will be returned after calculation: a small P-value indicated that differential genes incur enrichment in that TF item. Next, we counted the intersection of co-expressing coding gene sets of lncRNAs and target gene sets of transcription factor/ chromatin-regulated compounds, assessing the enrichment degree of the intersection by hypergeometric distribution, and obtaining the transcription factor obviously correlated to the IncRNAs to detect the transcription factors/chromatinregulated factors probably jointly exerting regulating effect with IncRNAs. Finally, visible network diagrams based on the analysis result of hypergeometric distribution were drawn.

Statistical analysis. The paired sample t-test was used to compare the expression level of lncRNAs. PCCs were calculated to evaluate the correlations between the expression level of lncRNAs and mRNAs. A 2-tailed Student's t-test was applied to compare the data on clinicopathological characteristics. The survival with log-rank score to examine the statistical significance was assessed using Kaplan-Meier analysis. The relative risk was evaluated using the multivariate Cox regression model, and hazard ratios with $95 \%$ confidence intervals were quantified to calculate the results. All statistical tests were analyzed with SPSS 17.0 System (SPSS, Chicago, IL, USA). P-value $<0.05$ was considered having statistical significance.

\section{Results}

IncRNA expression profile of ESCC. By comparing the lncRNA expression profiles in ESCC tissues and paired non-cancerous tissues, we acquired hundreds of differentially expressed lncRNAs from 3 patients with ESCC. The criteria of significant differential lncRNAs expression were defined as the absolute fold change (FC) value $>2.0$ and the $\mathrm{P}$-value $<0.05$. By the criteria mentioned above, the microarray results displayed that 182 lncRNAs, 106 upregulated and 76 downregulated, were significantly changed in ESCC tissues compared with paired non-cancerous tissues. The most upregulated lncRNAs were NONHSAT104436, ENST00000539535, ENST00000589379, NONHSAT023881 and ENST00000598376, of which NONHSAT104436 showed the largest upregulation (absolute FC, 27.25). The most downregulated lncRNAs were ENST00000530190, NONHSAT047224, ENST00000480669, NONHSAT142201 and NONHSAT083762, of which ENST00000530190 showed the largest downregulation (absolute FC, 17.88). The top 20 up- and downregulated lncRNAs are listed in Table II. The hierarchical clustering of the different expression lncRNAs among specimens were demonstrated in the heat map (Fig. 1A). By experimental experience, accurate and effective results of PCR verification were more likely obtained for lncRNAs with absolute value of $\mathrm{FC}>8$. Therefore, we chose 10 significant differentially expressed lncRNAs randomly from microarray results (absolute $\mathrm{FC}>8$ ) to validate using qRT-PCR (Fig. 1B). These data suggest that a range of lncRNA expression abnormalities in ESCC could be involved in the occurrence of ESCC.

LncRNA-mRNA coexpression profiles and the lncRNA function annotation. In order to investigate the function of lncRNAs with significant differential expression in ESCC, we mapped the IncRNA-mRNA coexpression pattern by calculating the PCCs of each lncRNA and mRNA expression value. Each IncRNA was found to be correlated with a set of mRNAs. As the file was too large, we selected NONHSAT104436 from the 182 significantly differentially expressed lncRNAs as a representative to explore the function of IncRNAs. NONHSAT104436 showed the highest upregulated lncRNAs (absolute FC, 27.25) among ESCC tissues versus paired non-carcinoma tissues. As the standard of absolute PCCs value $>0.8$, a total of 1,969 genes (e.g., SOX2) were related to IncRNA NONHSAT104436. The top 

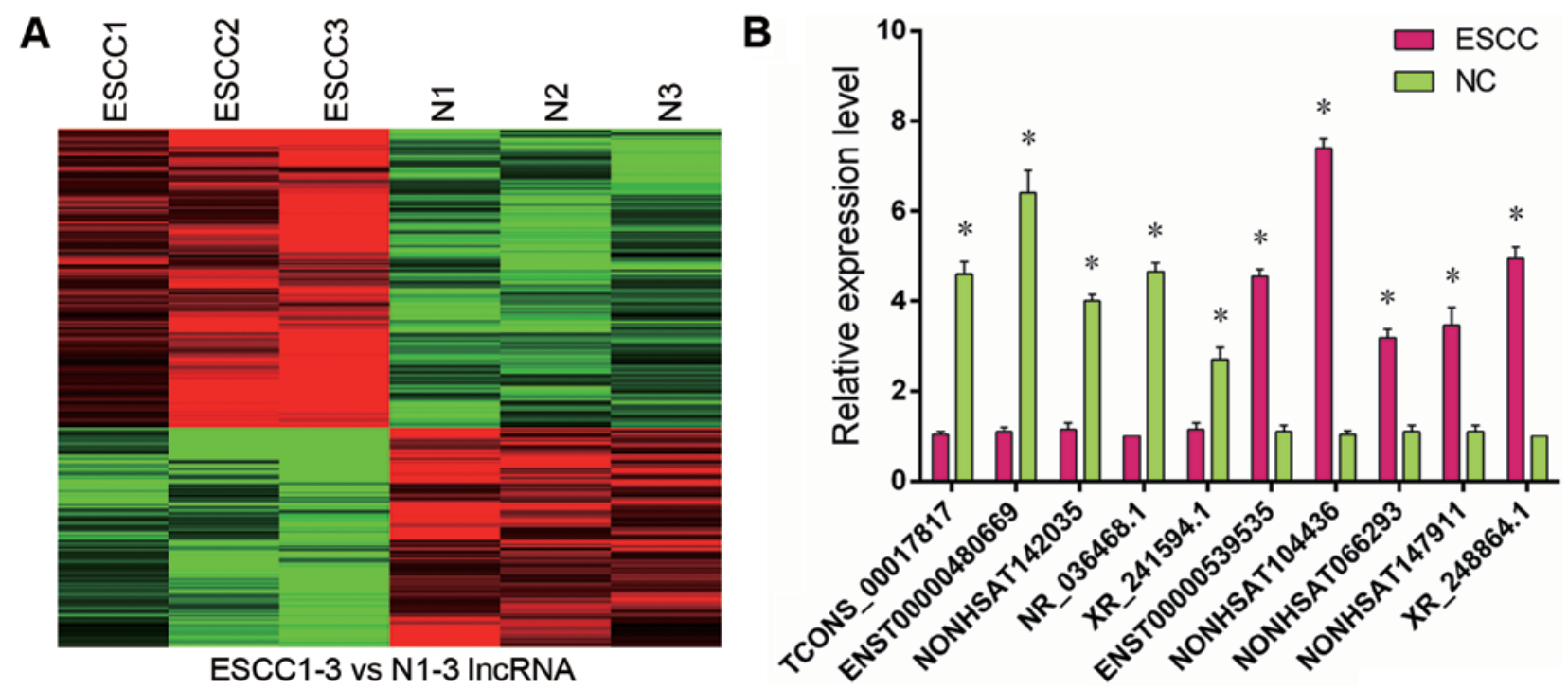

Figure 1. IncRNA expression profile. (A) Heat map was performed to demonstrate hierarchical clustering of the aberrant lncRNA expression pattern among specimens. (B) The microarray results were validated by qRT-PCR.

Table II. The top 20 up- and downregulated IncRNAs in ESCC compared with non-cancerous tissues.

\begin{tabular}{|c|c|c|c|}
\hline \multicolumn{2}{|c|}{ Upregulated in ESCC } & \multicolumn{2}{|c|}{ Downregulated in ESCC } \\
\hline $\begin{array}{l}\text { lncRNA } \\
\text { (Database_ID) }\end{array}$ & $\begin{array}{l}\text { Fold } \\
\text { change }\end{array}$ & $\begin{array}{c}\text { lncRNA } \\
\text { (Database_ID) }\end{array}$ & $\begin{array}{l}\text { Fold } \\
\text { change }\end{array}$ \\
\hline NONHSAT104436 & 27.25 & ENST00000530190 & 17.88 \\
\hline ENST00000539535 & 16.27 & NONHSAT047224 & 17.57 \\
\hline ENST00000589379 & 14.70 & ENST00000480669 & 17.06 \\
\hline NONHSAT023881 & 13.43 & NONHSAT142201 & 16.37 \\
\hline ENST00000598376 & 13.38 & NONHSAT083762 & 16.09 \\
\hline NONHSAT092291 & 20 & NONHSAT & 15.95 \\
\hline NONHSAT132869 & 12.14 & NONHSAT066087 & 15.55 \\
\hline NONHSAT115190 & 12.08 & NONHSAT067015 & 15.52 \\
\hline NONHSAT126998 & 12.98 & NONHSAT142102 & 15.50 \\
\hline ENST00000418557 & 12.77 & NONHSAT131584 & 15.44 \\
\hline ENST00000563101 & 12.66 & ENST00000434250 & 15.17 \\
\hline NONHSAT091534 & 11.65 & NONHSAT075654 & 14.70 \\
\hline NONHSAT015779 & 11.53 & NONHSAT083768 & 14.53 \\
\hline NONHSAT112918 & 11.40 & NONHSAT145733 & 14.25 \\
\hline NONHSAT103724 & 11.39 & NONHSAT059180 & 14.01 \\
\hline NONHSAT114324 & 10.33 & NONHSAT083765 & 13.67 \\
\hline NONHSAT119766 & 10.31 & NONHSAT146083 & 13.66 \\
\hline NONHSAT121426 & 10.30 & NONHSAT003383 & 13.64 \\
\hline & & ENST00000605056 & 13.51 \\
\hline NONHSAT015383 & 10.18 & NONHSAT015272 & 9.86 \\
\hline
\end{tabular}

20 genes are listed in Table III. Further, GO and KEGG pathways were applied to annotate the lncRNA NONHSAT104436 co-expressed mRNA function. Altogether 370 enrichment GO terms were acquired. By the ranks of enrichment, the top 20 reliably predicted terms from GO analysis are listed
Table III. The top 20 co-expressed genes of lncRNA NONHSAT 104436.

\begin{tabular}{lcc}
\hline Gene symbol & Corelation & P-value \\
\hline SOX2 & -0.988169796 & 0.000209103 \\
LARP7 & 0.98941143 & 0.000167583 \\
PRMT2 & -0.988028411 & 0.000214121 \\
C1orf159 & 0.986012133 & 0.000292122 \\
GLIPR1 & 0.985335534 & 0.000320993 \\
TSSK2 & -0.98506196 & 0.000333051 \\
CATSPER3 & 0.984998918 & 0.000335861 \\
GULP1 & -0.98493821 & 0.000338578 \\
MS4A2 & -0.9847261 & 0.000348156 \\
SPTY2D1 & -0.98417941 & 0.000373457 \\
LOC390660 & 0.98307808 & 0.000427104 \\
RAB11B & -0.982256526 & 0.000469453 \\
SLC9A11 & -0.982017597 & 0.000482143 \\
SNX3 & -0.981356548 & 0.000518127 \\
FGL1 & 0.980857587 & 0.000546141 \\
ZNF773 & 0.980597985 & 0.000561005 \\
CD34 & -0.980127267 & 0.000588464 \\
SIRT2 & -0.979508095 & 0.000625575 \\
SLC25A26 & -0.978628587 & 0.000680225 \\
ATF4 & 0.978557598 & 0.000684736 \\
\hline
\end{tabular}

in Table IV. It indicates that the significantly enriched GO terms were involved in structural constituent of ribosome, protein binding, angiotensin maturation, regulation of cellular amino acid metabolic process and cytosolic large ribosomal subunit. Moreover, the KEGG pathways analysis results are listed in Table V, including ribosome, proteasome, glyoxylate and dicarboxylate metabolism, RNA degradation and arginine and proline metabolism. 
Table IV. The top 20 GO analysis enrichment terms of lncRNA NONHSAT104436.

\begin{tabular}{|c|c|c|c|}
\hline Enrichment term & Description & List hits & P-value \\
\hline GO:0003735 & Structural constituent of ribosome & 27 & 0.000119393 \\
\hline GO:0005515 & Protein binding & 577 & 0.000184285 \\
\hline GO:0002003 & Angiotensin maturation & 6 & 0.000196547 \\
\hline GO:0006521 & Regulation of cellular amino acid metabolic process & 13 & 0.00020788 \\
\hline GO:0022625 & Cytosolic large ribosomal subunit & 13 & 0.000256284 \\
\hline GO:0008083 & Growth factor activity & 27 & 0.000353119 \\
\hline GO:0006412 & Translation & 37 & 0.000372073 \\
\hline GO:0001916 & Positive regulation of $\mathrm{T}$ cell mediated cytotoxicity & 7 & 0.000383209 \\
\hline GO:0005178 & Integrin binding & 19 & 0.00046843 \\
\hline GO:0012507 & ER to Golgi transport vesicle membrane & 10 & 0.000528766 \\
\hline GO:0031290 & Retinal ganglion cell axon guidance & 7 & 0.000563342 \\
\hline GO:0002486 & $\begin{array}{l}\text { Antigen processing and presentation of endogenous peptide } \\
\text { antigen via MHC class I via ER pathway, TAP-independent }\end{array}$ & 3 & 0.000576515 \\
\hline GO:0046977 & TAP binding & 3 & 0.000576515 \\
\hline GO:0061146 & Peyer's patch morphogenesis & 3 & 0.000576515 \\
\hline GO:0061574 & ASAP complex & 3 & 0.000576515 \\
\hline GO:0072011 & Glomerular endothelium development & 3 & 0.000576515 \\
\hline GO:0060306 & Regulation of membrane repolarization & 5 & 0.000702145 \\
\hline GO:0060333 & Interferon- $\gamma$-mediated signaling pathway & 14 & 0.000776854 \\
\hline GO:0016597 & Amino acid binding & 7 & 0.000804767 \\
\hline GO:0071353 & Cellular response to interleukin-4 & 7 & 0.000804767 \\
\hline
\end{tabular}

Table V. KEGG pathway analysis of lncRNA NONHSAT104436 co-expressed genes.

\begin{tabular}{llrr}
\hline Enrichment term & \multicolumn{1}{c}{ Description } & List hits & P-value \\
\hline path:hsa03010 & Ribosome & 25 & 0.0003345 \\
path:hsa03050 & Proteasome & 12 & 0.000374178 \\
path:hsa00630 & Glyoxylate and dicarboxylate metabolism & 7 & 0.00537185 \\
path:hsa03018 & RNA degradation & 14 & 0.005778636 \\
path:hsa00330 & Arginine and proline metabolism & 12 & 0.00747148 \\
path:hsa00640 & Propanoate metabolism & 7 & 0.010434297 \\
path:hsa03013 & RNA transport & 24 & 0.011041138 \\
path:hsa00250 & Alanine, aspartate and glutamate metabolism & 8 & 0.011102205 \\
path:hsa04614 & Renin-angiotensin system & 5 & 0.014622776 \\
path:hsa00380 & Tryptophan metabolism & 8 & 0.024354511 \\
path:hsa03015 & mRNA surveillance pathway & 14 & 0.032809394 \\
path:hsa05216 & Thyroid cancer & 6 & 0.041797251 \\
path:hsa00471 & D-glutamine and D-glutamate metabolism & 2 & 0.043217651 \\
path:hsa04144 & Endocytosis & 27 & 0.043796016
\end{tabular}

Then, the whole set of significantly differentially expressed lncRNA co-expression mRNAs were also annotated by applying GO and KEGG pathway analysis. Based on enrichment ranks, the top 200 and 500 reliably predicted terms from GO and KEGG pathway analysis were selected, respectively (Fig. 2). In our survey (Fig. 2A and $\mathrm{B}$ ), the GO analysis showed that the enrichment terms in biological process mainly included TAP-independent, cell differentiation, collagen fibril organization, cell proliferation and cell adhesion. With respect to cellular components (Fig. 2C and D), the significant enrichment terms connect with differentially expressed lncRNAs mainly included nuclear chromosome, condensed chromosome kinetochore, U4 snRNP, MCM complex and proteasome core complex. 
A

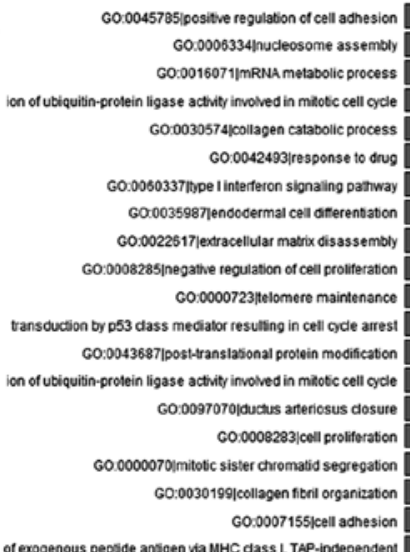

C GO:0043234|protein complex sicle membrane Co:0042612/MHC dass I protein complex 60:00319011earty endosome membrane 0 00000000 nelanosome G0:0000776||vinetochore 60:0000785/chromatin 60:0005876|spincle microtubule GO-0030496|miabody 60:0005730|nucleolus G0:0005813/centrosome G0:0000775/chromosome, centromeric region G0.0005839proteasome core complex 60:0042555IMCM complex G0.0005687|U4 SnRNP 60:0000777|condensed chromosome kinetochore co:0000228Inudear chromosome

E c04714|transmembrane receptor protein trosine kinase advivt G0:0005178/integrin binding G0.0005102/;0coptor binding G0:0019899/enzame binding Go:0017048jReno GTPase Dinan G0:0005096|GTPase activator activity G0.0042802|identical protein binding G00016887/aTpase activty C0.0046977T/AP binding G0:00020201protease binding 60:0008201||heparin bindin G0.0042803: protein homodimerization adtivty 60:0003577 Toma binang c0:0048407|platelelt-derived growe factor binaing G0.003067eiRac guanh-nudeotde exchange fador activity G0:0005092]GDP-dissociabon innibior activet 60:00440221001/(A) RNA Dinding 60:0003779|actin binang 60:0070182|DNA polymerase binding G0.0005524/4TP bindin
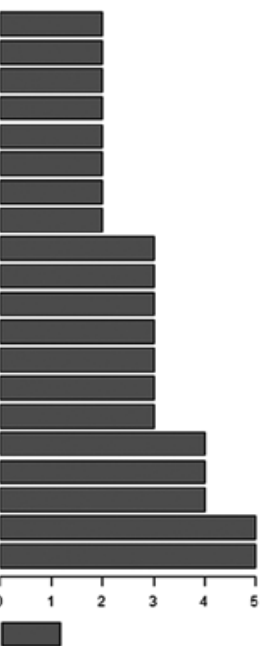

尚
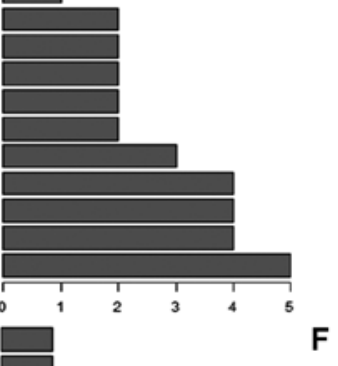

G

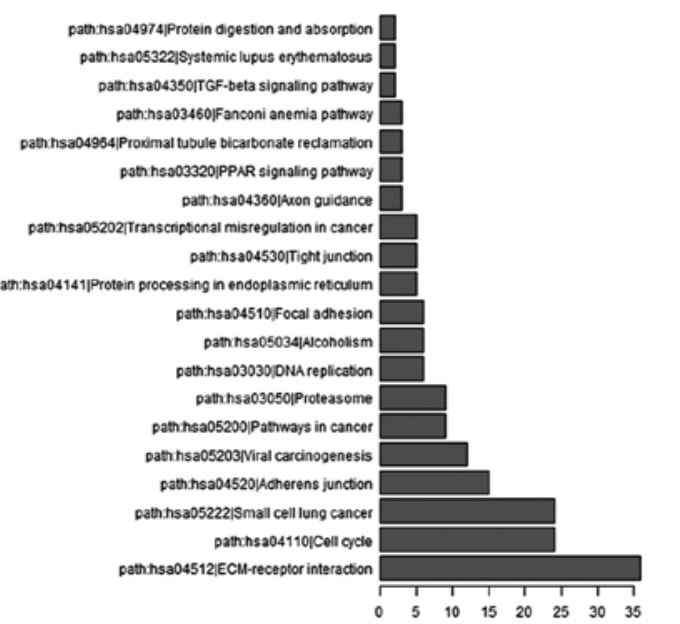

B

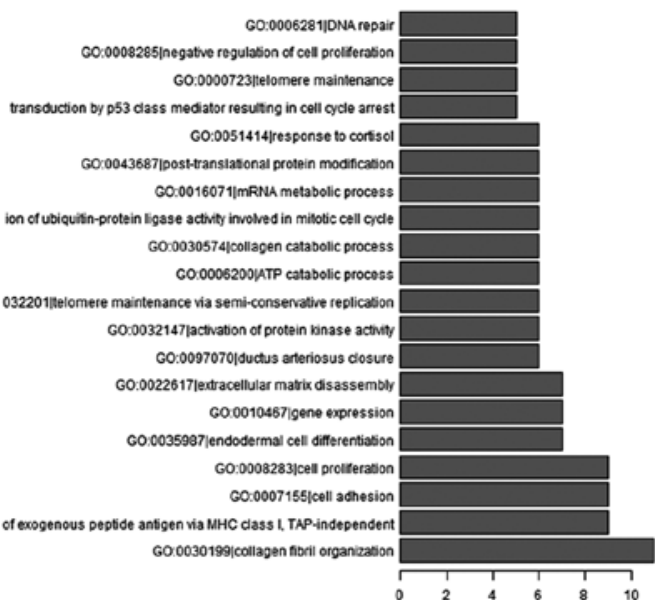

D

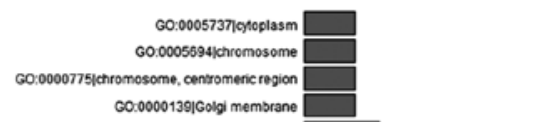
Go.0043234|crotein complex G0.0005925Focal achesion

co.0005783/endoplagmic retculum 60:000507øssinale microtusule Go.0005s3annudeus co:0030496|midboody

60.0005613/centrosome Go.0015629|actin chosketeton G0:0031012|extracollular mastix 60:0042470/melanosome GO:0000777|cendensed chromosome kinetochore 60.0005839proteas ome core complex 60:00007es/chromatin 60.0000228inudear chromosome G0:0042555IMCM complex GO.0005637N4 InRNP

60:0008083!growent factor actovet co:0004674|protein serine/greeonine knase activaty G0-0005391/sodium potassium-exchanging ATPase actvity

C0:0003677|10Na binding 60.0005518|collagen binding 60.0003735|studural consttuvent of ribosome 60:00082011heparin binding

G0.0046982|protein heterodimerization advivit Go:0030676|Rac guany-hude otbde exchange fador actvet G0.0005092/GOP-dissociacon inhibtor actvit GO:0042803|protein nomodimerizaton actviny G0.0016887/4TPase advinty G0.0046977/TAP binding G0:00170481RRo GTPase binding 60.0048407|platelet-derived growen factor binding 60:0003779adtin bindin G0.0042802identical protein binding GO:0070182|DNA potymerase binding Go.0005524/ATP binding G0.0044822:poly(A) Rave bindin

H

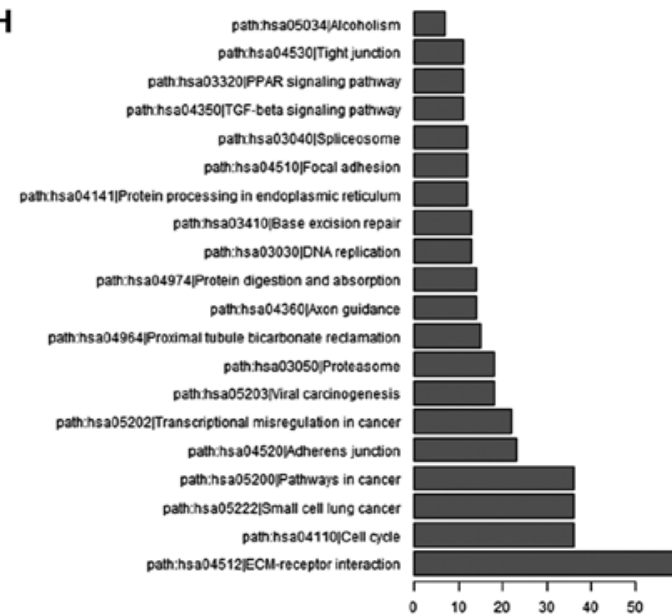

Figure 2. GO and pathway analysis. (A and B) Biological process of top 200 and $500 \mathrm{GO}$ terms. (C and D) Cellular component of top 200 and $500 \mathrm{GO}$ terms. (E and F) Molecular function of top 200 and 500 GO terms. (G and H) The top 200 and 500 pathway analysis. 

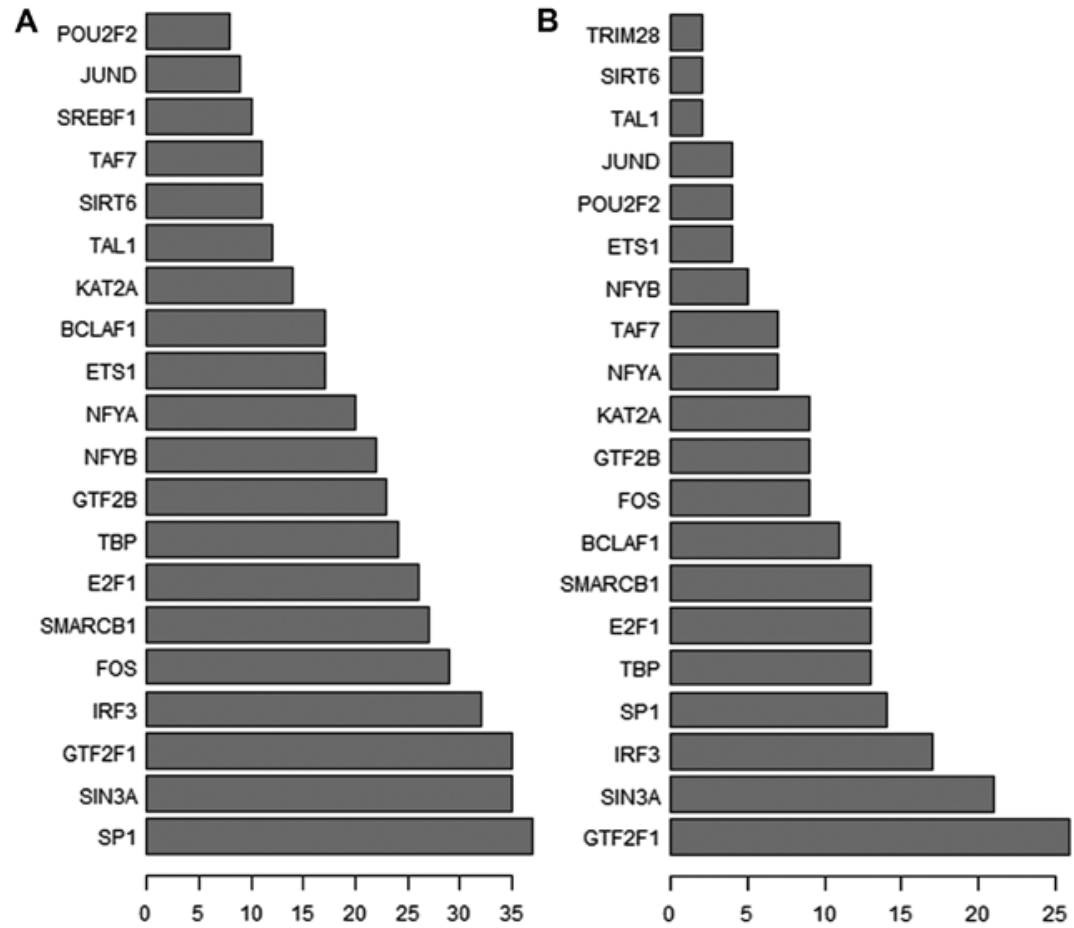

Figure 3. TFs analysis for the aberrant lncRNA co-expression genes. (A) The significant TFs of the top 200 enrichment terms. (B) The significant TFs of the top 500 enrichment terms.

Finally, ATP binding, DNA polymerase binding, actin binding, poly(A)RNA binding and platelet-derived growth factor binding were involved in the significant enriched molecular function (Fig. 2E and F). Likewise, the KEGG pathway analysis showed that the top five enrichment terms (Fig. 2G and H) were ECM-receptor interaction, cell cycle, small cell lung cancer, viral carcinogenesis, pathway in cancer.

Analysis of cis regulatory mRNAs of the aberrant lncRNAs. In order to predict the potential 'cis-regulated mRNAs' of IncRNAs, we identified the same locus co-expressed genes within $300 \mathrm{kbp}$ windows of the significantly differentially expressed lncRNAs. Based on the absolute PCCs value $>0.8$ and $\mathrm{P}$-value $<0.05$, the results of the cis prediction analysis are listed in Table VI. It included 50 significantly differently expressed lncRNAs and 67 different mRNAs. Because some genes had two or even more transcripts, the 50 lncRNAs had 85 cis genes (Table VI). Among these, lncRNA TCONS_00012018 had 5 cis genes, and two lncRNAs (NONHSAT119511 and TCONS_00017817) had 3 cis genes. VEGFA and WISP1 were the cis genes of the aberrantly expressed lncRNAs NONHSAT112918 and NR_037944.1, respectively.

Analysis of trans regulatory $m R N A$ s of the aberrant lncRNAs. Transcription factors could mediate chromatin regulation and transcription, which interact with many lncRNAs. Therefore, trans regulatory mechanism could be another useful indicator to predict lncRNA-target genes. In this study, differentially expressed IncRNAs co-expressed coding genes enrichment in TFs terms demonstrated that a total of 168 1ncRNAs were regulated by 95 TFs. Next, we selected the top 200 and top 500 according to predicted reliability rank in previously mentioned relation of 'IncRNA-TF', recording the frequency of each TF and summarizing those TFs with a great number of functional annotations to reflect the overall function distribution of the differentially expressed IncRNAs (Fig. 3). As demonstrated in Fig. 3, these lncRNAs may be mostly regulated by the 20 TFs. As each lncRNA could secure one to many TF-lncRNA relation groups, the network of TF-lncRNA is too large. Therefore, we took the top 100 regulating relations based on P-value to draw binary-relation network diagrams using Cytoscape software (Fig. 4). In Fig. 4, 56 lncRNAs and $20 \mathrm{TFs}$ were involved, and the transcription factors GTF2F1, SIN3A and IRF3 were the most invloved, which modulate 13, 13, and 11 lncRNAs, respectively. Considering IncRNA-TF was derived from the enrichment of various genes, we drew ternary-relation network diagrams on account of the top 300 IncRNA-genes and TF-genes relation groups to reflect the relationship of TFs, lncRNAs and target genes (Fig. 5). It includes 3 upregulated lncRNAs (NONHSAT126998, TCONS_00006172 and XR_245796.1) and 1 downregulated lncRNA (XR_245796.1), 3 TFs (BCLAF1, GTF2F1 and SIN3A) and 124 target genes in this map.

Target gene predictions. The regulatory roles of lncRNA on target genes were mediated by $c i s$ - and trans-regulatory mechanisms $(22,23)$. In this study, the cis analysis indicated 50 lncRNAs regulated 85 mRNAs (Table VI). Among these target genes, biological function of VEGFA and WISP1 have been reported in ESCC, and their paired lncRNAs were NONHSAT112918 and NR_037944.1, respectively. Considering the trans-regulatory mechanisms, differentially expressed IncRNAs co-expressed mRNA enrichment in TFs terms indicated that lncRNAs may be mostly regulated by the 
Table VI. LncRNAs and their cis genes in the chromosome.

Target ID $\begin{gathered}\text { Fold } \\ \text { change }\end{gathered}$ Gene symbol Corelation

\begin{tabular}{|c|c|c|c|}
\hline 00434250 & 15.17 & PHYHD1 & 0.847491793 \\
\hline ENST00000434250 & 2.31 & DOLPP1 & -0.844775134 \\
\hline ENST00000480669 & 17.06 & MYNN & -0.922763789 \\
\hline ENST00000480669 & 3.66 & MYNN & -0.853609445 \\
\hline FR331033 & 2.09 & EFNB1 & 0.986834594 \\
\hline NONHSAG001208 & 2.03 & PPCS & 0.91715456 \\
\hline NONHSAG008446 & 2.65 & TMEM132A & 0.94221026 \\
\hline NONHSAG008446 & 2.65 & MS4A18 & -0.839282644 \\
\hline NONHSAG008447 & 2.22 & TMEM132A & 0.977419933 \\
\hline NONHSAG008447 & 2.22 & TMEM109 & -0.873421131 \\
\hline NONHSAG024488 & 5.19 & NFIC & 0.839314642 \\
\hline NONHSAG024488 & 5.19 & TJP3 & 0.826013387 \\
\hline NONHSAG024488 & 5.19 & FZR1 & -0.818436791 \\
\hline NONHSAG030248 & 2.15 & STRADB & 0.945685017 \\
\hline NONHSAG030248 & 2.15 & STRADB & 0.891826712 \\
\hline NONHSAG047728 & 2.24 & LOC10050 & 0.853688706 \\
\hline NONHSAG052739 & 2.35 & CTSL1 & 0.936597756 \\
\hline NONHSAT003287 & 2.11 & PODN & 0.983466994 \\
\hline NONHSAT003383 & 10.18 & ACOT11 & 0.987214284 \\
\hline NONHSAT003383 & 6.11 & $\mathrm{ACC}$ & 1246 \\
\hline NONHSAT003383 & 6.11 & C1orf177 & 0.830504414 \\
\hline NONHSAT010549 & 7.02 & KMO & 5256 \\
\hline NONHSAT013915 & 2.11 & DDIT4 & 0.935602917 \\
\hline NONHSAT015779 & 11.53 & ENTPD1 & 0.936 \\
\hline NONHSAT016005 & 2.3 & FAM178A & 0.898055251 \\
\hline NONHSAT018044 & 2.05 & MICAL2 & 0.934861434 \\
\hline NONHSAT023402 & 3.03 & ANKRD42 & 0.931194591 \\
\hline NONHSAT023402 & 3.03 & ANKRD42 & 0.871767285 \\
\hline NONHSAT028105 & 2.14 & SPATS2 & 0.92440566 \\
\hline NONHSAT028105 & 2.14 & TROAP & 0.833886125 \\
\hline NONHSAT028874 & 2.16 & NAB2 & 0.985654077 \\
\hline NONHSAT037520 & 4.532 & GALNTL1 & 0.898048372 \\
\hline NONHSAT042059 & 2.18 & CCNDBP1 & 0.991255862 \\
\hline NONHSAT042059 & 2.18 & ADAL & 0.853427049 \\
\hline NONHSAT042059 & 2.18 & TUBGCP4 & -0.826893233 \\
\hline NONHSAT042184 & 7.01 & B2M & 0.981064031 \\
\hline NONHSAT051867 & 2.30 & RAB11FIP3 & 0.828468513 \\
\hline NONHSAT051867 & 2.30 & PIGQ & 0.821774805 \\
\hline NONHSAT066040 & 2.12 & ZNF568 & -0.82107802 \\
\hline NONHSAT066087 & 15.55 & ZNF570 & 0.938002312 \\
\hline NONHSAT066293 & 8.76 & PLEKH & 0.988420647 \\
\hline NONHSAT066293 & 8.76 & PLEKHG2 & 0.988089203 \\
\hline NONHSAT066293 & 8.76 & NCCRP1 & -0.83203032 \\
\hline NONHSAT066293 & 8.76 & DLL3 & 0.81520705 \\
\hline NONHSAT076108 & 2.08 & GLS & 0.978591053 \\
\hline NONHSAT076120 & 2.36 & MYO & 0.942125167 \\
\hline NONHSAT081970 & 15.95 & DOPEY2 & 0.890012356 \\
\hline NONHSAT081970 & 2.70 & CBR3 & 0.842388559 \\
\hline
\end{tabular}

Table VI. Continued.

Target ID $\begin{gathered}\text { Fold Gene symbol Corelation } \\ \text { change }\end{gathered}$

\begin{tabular}{lrll}
\hline NONHSAT083006 & 2.19 & COL6A1 & 0.876584355 \\
NONHSAT083006 & 2.19 & COL6A2 & 0.876358371 \\
NONHSAT090846 & 2.19 & COL8A1 & 0.873385734 \\
NONHSAT112918 & 11.40 & VEGFA & 0.91993667 \\
NONHSAT115190 & 12.08 & TNFAIP3 & 0.959731249 \\
NONHSAT119551 & 4.70 & C7orf46 & 0.971787358 \\
NONHSAT119551 & 4.70 & C7orf46 & 0.95260555 \\
NONHSAT119551 & 4.70 & C7orf46 & 0.937119124 \\
NONHSAT119766 & 10.31 & CREB5 & 0.909097249 \\
NONHSAT130117 & 2.23 & MLANA & 0.890075793 \\
NONHSAT132869 & 12.14 & CTSL1 & 0.985462012 \\
NONHSAT143438 & 2.23 & IL34 & 0.973354259 \\
NONHSAT147911 & 9.79 & TNFRSF12A & 0.989875868 \\
NONHSAT147911 & 9.79 & THOC6 & 0.967139208 \\
NONHSAT147911 & 9.79 & IL32 & 0.9631379 \\
NONHSAT147911 & 9.79 & PAQR4 & 0.918730586 \\
NONHSAT147911 & 9.79 & KREMEN2 & 0.816564421 \\
NR_024341.1 & 2.21 & CACNA1B & 0.915462971 \\
NR_024341.1 & 2.21 & CACNA1B & -0.845942556 \\
NR_036468.1 & 9.52 & CIDEA & 0.948597107 \\
NR_036468.1 & 9.52 & SLMO1 & -0.942484643 \\
NR_036468.1 & 9.52 & IMPA2 & 0.832340155 \\
NR_036468.1 & 9.52 & IMPA2 & 0.825982055 \\
NR_037944.1 & 3.77 & WISP1 & 0.975128047 \\
NR_073516.1 & 2.49 & CPNE7 & 0.849947236 \\
TCONS_00006172 & 7.43 & P39195 & 0.881950723 \\
TCONS_00012018 & 2.67 & MLLT4 & 0.916817949 \\
TCONS_00012018 & 2.67 & MLLT4 & 0.916084696 \\
TCONS_00012018 & 2.67 & MLLT4 & 0.853744868 \\
TCONS_00012018 & 2.67 & MLLT4 & 0.842280628 \\
TCONS_00012018 & 2.67 & MLLT4 & 0.826596987 \\
TCONS_00014231 & 2.06 & ZNF862 & -0.818149074 \\
TCONS_00017817 & 8.09 & BMS1 & 0.95552454 \\
TCONS_00017817 & 8.09 & BMS1 & 0.948137275 \\
TCONS_00017817 & 8.09 & BMS1 & 0.88226429 \\
TCONS_00024250 & 8.95 & LOC729264 & 0.859340357 \\
TCONS_12_00008392 & 2.18 & LOC642311 & 0.948471492 \\
\hline & & & \\
NOF & & &
\end{tabular}

$20 \mathrm{TFs}$ (Fig. 3). It has been reported in the literature that these TFs SP1, E2F1 and BCLAF1 were related with ESCC development (24-26). Analyzing the relationship between IncRNAs and TFs, we found that the three TFs closely related to 22 lncRNAs, and which indicated that they may have important function in ESCC. To further explore potential biological roles of lncRNAs in ESCC, we selected lncRNAs based on FC value $>10$ and lncRNA-mRNA co-expression analysis PCCs $>0.90$. The co-expressed coding genes should accord with GO and KEGG terms enrichment such as cell differentiation, ECM-receptor interaction, cell proliferation, pathway in cancer. Their target genes biological functions had been reported in ESCC in numerous cases. Ultimately, we selected 


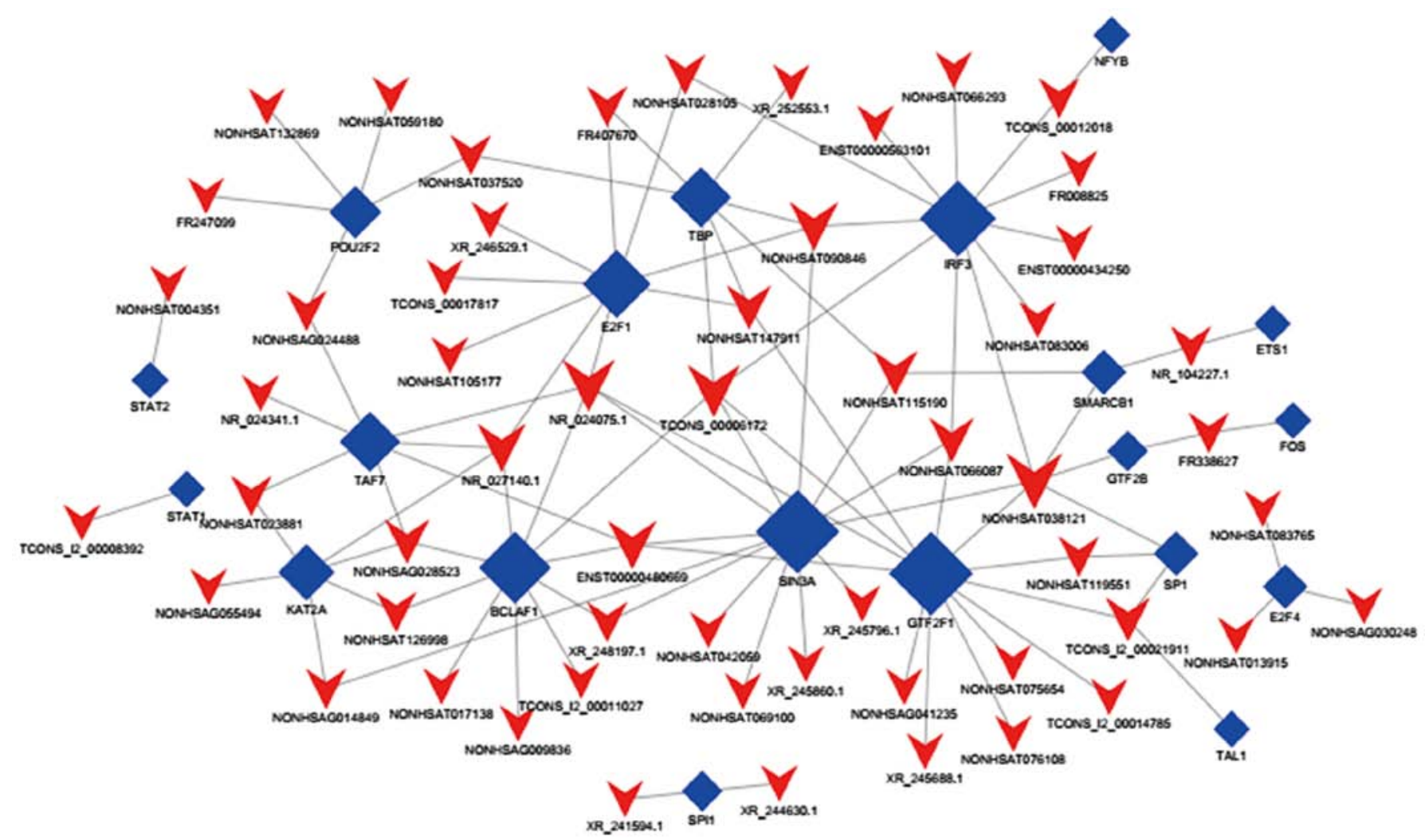

Figure 4. LncRNA-TFs analysis. Network of the top 100 regulating relations of LncRNA-TFs (consist of 56 LncRNAs and 20 TFs).

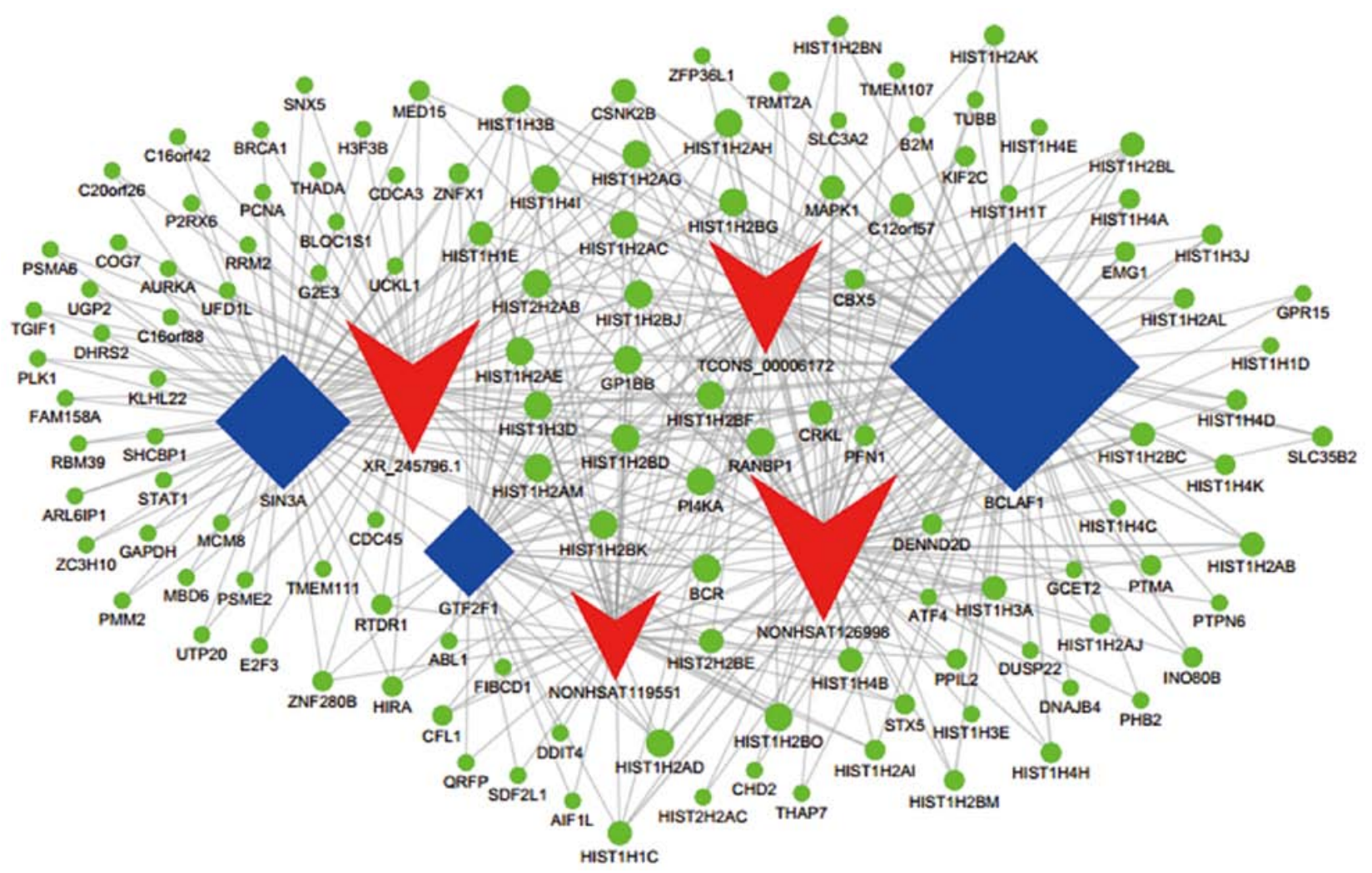

Figure 5. TF-lncRNA-target genes. Network of the top 300 regulating relations of TF-lncRNA-target genes (consist of 4 lncRNAs, 3 TFs and 124 target genes). 


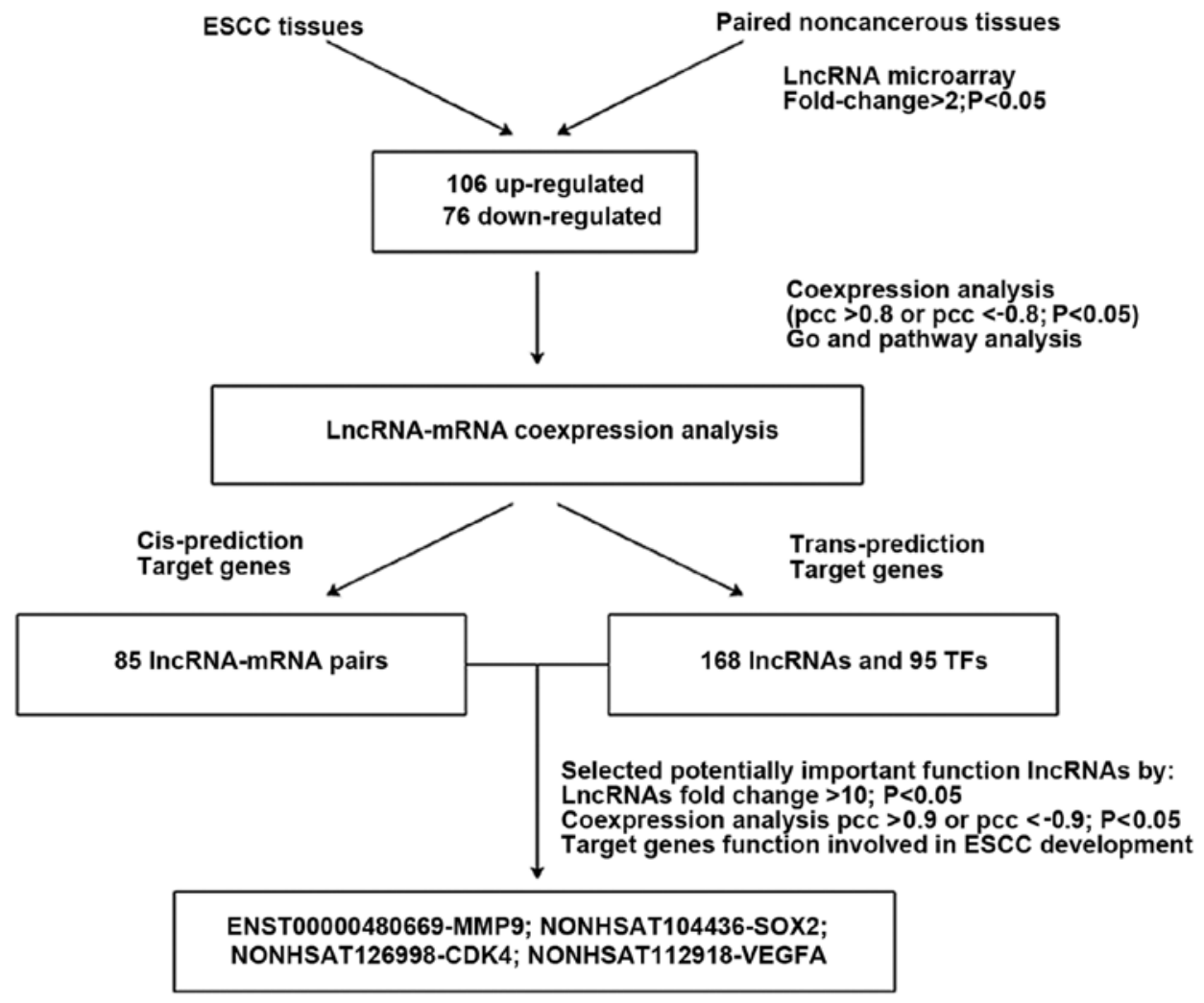

Figure 6. Filtering procedure. The overview of the process for filtering lncRNAs in ESCC tissues and matched non-carcinoma tissues.
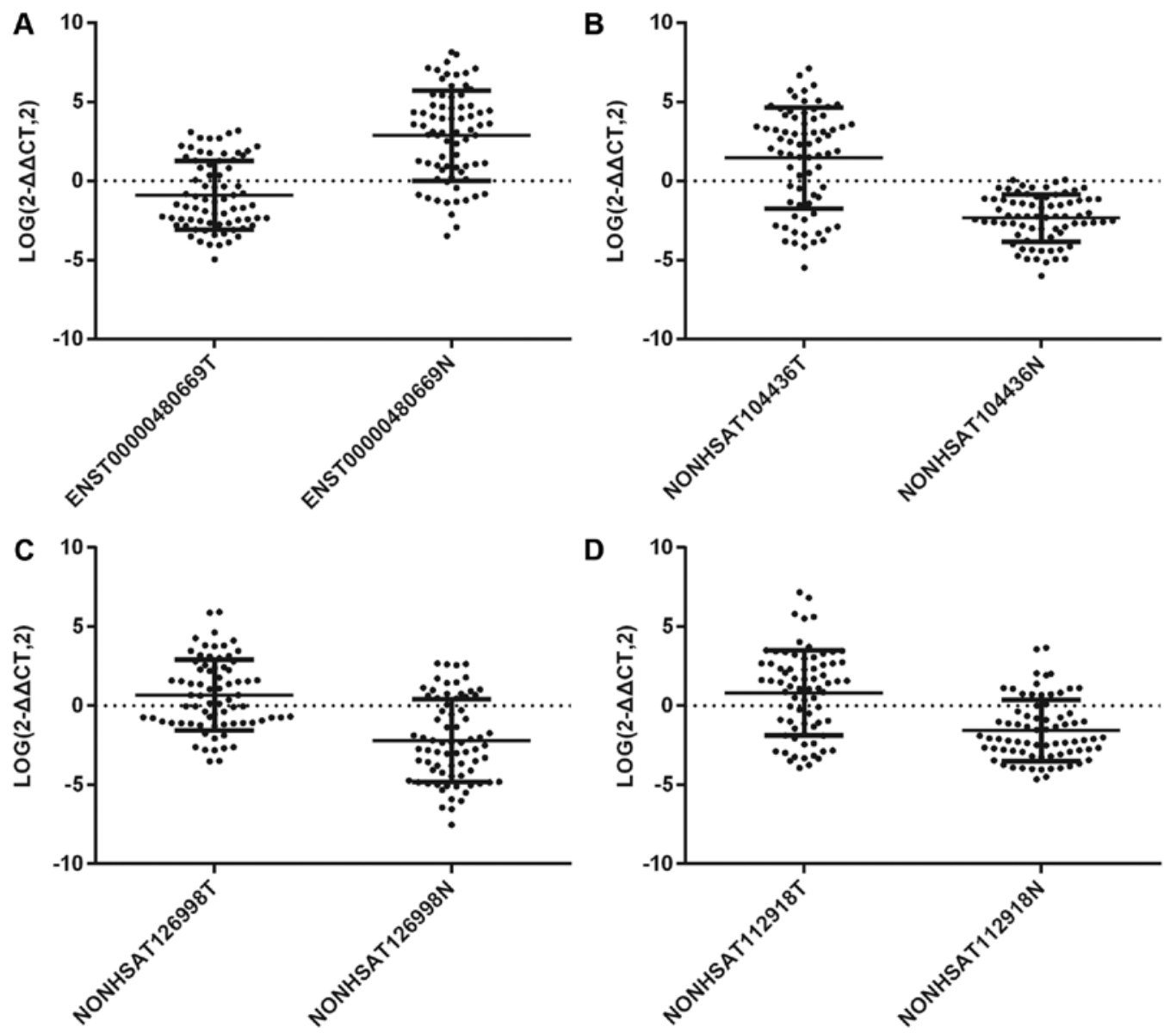

Figure 7. QRT-PCR analysis of the selected four lncRNAs. (A) ENST00000480669 was downregulated. (B) NONHSAT104436 was upregulated. (C) NONHSAT126998 was upregulated. (D) NONHSAT112918 was upregulated (all P-values $<0.05$ ). 
Table VII. The relationship between clinical features and the expression level of IncRNA ENST00000480669 and NONHSAT104436 in 73 patients with ESCC.

Variable N $\frac{\text { ENST00000480669 }}{\text { Mean } \pm \text { SD P-value }} \frac{\text { NONHSAT104436 }}{\text { Mean } \pm \text { SD P-value }}$

Age (years)

$\begin{array}{llllll}\geq 60 & 43 & -0.94 \pm 2.15 & 0.863 & 1.51 \pm 3.05 & 0.815 \\ <60 & 30 & -0.85 \pm 2.25 & & 1.33 \pm 3.42 & \end{array}$

Gender

$\begin{array}{llllll}\text { Male } & 55 & -0.81 \pm 2.14 & 0.506 & 1.74 \pm 3.03 & 0.156 \\ \text { Female } & 18 & -1.20 \pm 2.31 & & 0.51 \pm 3.53 & \end{array}$

Tumor size

$(\mathrm{cm})$

\begin{tabular}{llllll}
$\leq 5$ & 47 & $-0.89 \pm 2.28$ & 0.953 & $1.38 \pm 3.34$ & 0.837 \\
$>5$ & 26 & $-0.93 \pm 2.01$ & & $1.54 \pm 2.93$ & \\
T stage & & & & & \\
T1-2 & 24 & $-0.54 \pm 2.40$ & 0.318 & $0.51 \pm 3.12$ & 0.081 \\
T3-4 & 49 & $-1.08 \pm 2.06$ & & $1.89 \pm 3.14$ & \\
N stage & & & & & \\
N0 & 38 & $-0.37 \pm 2.36$ & 0.026 & $0.91 \pm 3.24$ & 0.141 \\
N1 & 35 & $-1.48 \pm 1.81$ & & $2.01 \pm 3.06$ & \\
M stage & & & & & \\
M0 & 62 & $-0.80 \pm 2.26$ & 0.221 & $1.03 \pm 3.12$ & 0.008 \\
M1 & 11 & $-1.50 \pm 1.56$ & & $3.76 \pm 2.56$ & \\
TNM stage & & & & & \\
I-II & 39 & $-0.50 \pm 2.37$ & 0.086 & $0.75 \pm 3.22$ & 0.046 \\
III-IV & 34 & $-1.37 \pm 1.86$ & & $2.23 \pm 2.99$ & \\
\hline
\end{tabular}

Association analysis of lncRNAs with clinicopathological characteristics. To better understand the roles of lncRNAs in ESCC, we first checked the expression levels of the 4 lncRNAs (ENST00000480669, NONHSAT104436, NONHSAT126998 and NONHSAT112918). The expression of lncRNAs were quantified via RT-PCR converting the $2^{-\Delta \Delta C t}$ to $\log \left(2^{-\Delta \Delta C t}\right)$ values in ESCC tissues and matched noncancerous tissues (Fig. 7). The data demonstrated that the expression pattern of the four selected IncRNAs analyzed by microarray was consistent with that done by RT-PCR. We next compared the expression levels of these genes with some specific clinicopathological characteristics. The results are listed in Tables VII and VIII. ENST00000480669 was significantly related to lymph node metastasis $(\mathrm{P}=0.026)$. NONHSAT104436 was significantly related to distant metastasis $(\mathrm{P}=0.008)$. NONHSAT126998 was significantly related to lymph node metastasis $(\mathrm{P}=0.010)$ and TNM stage $(\mathrm{P}=0.019)$. In addition, NONHSAT112918 was significantly related to tumor infiltrating stage $(\mathrm{P}=0.034)$.

Correlations between lncRNA expression and ESCC prognosis. Univariate survival analysis was used to evaluate the relationship between the lncRNA expression level and
Table VIII. The relationship between clinical features and the expression level of lncRNA NONHSAT126998 and NONHSAT112918 in 73 patients with ESCC.

\begin{tabular}{|c|c|c|c|c|c|}
\hline \multirow[b]{2}{*}{ Variable } & \multirow[b]{2}{*}{$\mathrm{N}$} & \multicolumn{2}{|c|}{ NONHSAT126998 } & \multicolumn{2}{|c|}{ NONHSAT112918 } \\
\hline & & Mean \pm SD & $\mathrm{P}$-value & Mean \pm SD & P-value \\
\hline \multicolumn{6}{|c|}{ Age (years) } \\
\hline$\geq 60$ & 43 & $0.85 \pm 2.73$ & 0.918 & $0.95 \pm 2.38$ & 0.229 \\
\hline$<60$ & 30 & $0.79 \pm 2.68$ & & $0.31 \pm 2.01$ & \\
\hline \multicolumn{6}{|l|}{ Gender } \\
\hline Male & 55 & $0.98 \pm 2.66$ & 0.384 & $0.68 \pm 2.37$ & 0.992 \\
\hline Female & 18 & $0.34 \pm 2.81$ & & $0.69 \pm 1.84$ & \\
\hline \multicolumn{6}{|c|}{$\begin{array}{l}\text { Tumor size } \\
(\mathrm{cm})\end{array}$} \\
\hline$\leq 5$ & 47 & $0.80 \pm 2.82$ & 0.894 & $0.51 \pm 1.96$ & 0.423 \\
\hline$>5$ & 26 & $0.88 \pm 2.50$ & & $1.00 \pm 2.69$ & \\
\hline \multicolumn{6}{|l|}{ T stage } \\
\hline $\mathrm{T} 1-2$ & 24 & $0.27 \pm 3.01$ & 0.221 & $0.13 \pm 2.12$ & 0.034 \\
\hline $\mathrm{T} 3-4$ & 49 & $1.10 \pm 2.51$ & & $1.36 \pm 2.27$ & \\
\hline \multicolumn{6}{|l|}{ N stage } \\
\hline No & 38 & $0.06 \pm 2.69$ & 0.010 & $0.87 \pm 2.04$ & 0.462 \\
\hline N1 & 35 & $1.66 \pm 2.47$ & & $0.48 \pm 2.45$ & \\
\hline \multicolumn{6}{|l|}{ M stage } \\
\hline M0 & 62 & $0.71 \pm 2.78$ & 0.387 & $0.73 \pm 2.22$ & 0.713 \\
\hline M1 & 11 & $1.48 \pm 2.08$ & & $0.45 \pm 2.47$ & \\
\hline \multicolumn{6}{|c|}{ TNM stage } \\
\hline I-II & 39 & $0.15 \pm 2.66$ & 0.019 & $0.72 \pm 2.07$ & 0.880 \\
\hline III-IV & 34 & $1.61 \pm 2.55$ & & $0.64 \pm 2.45$ & \\
\hline
\end{tabular}

cancer prognosis. There were no samples excluded from the univariate survival analysis during the three years of follow-up. The 3-year overall survival rate of 73 patients was $39.7 \%$. The outcome of statistical analysis showed that the cumulative overall survival rate was poor with high expression of NONHSAT104436 and NONHSAT126998. The 3 -year survival rate for ESCC patients with high expression of NONHSAT104436 was $25.5 \%$, whereas, the patients with NONHSAT104436 low expression had a 3-year survival rate of $65.4 \%$ ( $\mathrm{P}=0.003$, Fig. 8). The results of NONHSAT126998 were similar to that of NONHSAT104436. The patients with high expression of NONHSAT126998 had a poorer 3-year survival rate $(31.1 .0 \%)$ than the patients with low expression of NONHSAT126998 (53.6\%; P=0.032, Fig. 8). On the contrary, the findings of ENST00000480669 were different from NONHSAT104436 and NONHSAT126998, i.e., patients with high expression of ENST00000480669 gained a relatively higher 3-year survival rate $(53.8 \%)$ than patients with low expression of ENST00000480669 (31.9\%; P=0.040, Fig. 8). There was no statistical significance for the overall survival rate between high expression and low expression of NONHSAT112918 $(\mathrm{P}=0.374)$. Three-year survival rate for high and low expression was 33.3 and $41.7 \%$, respectively 

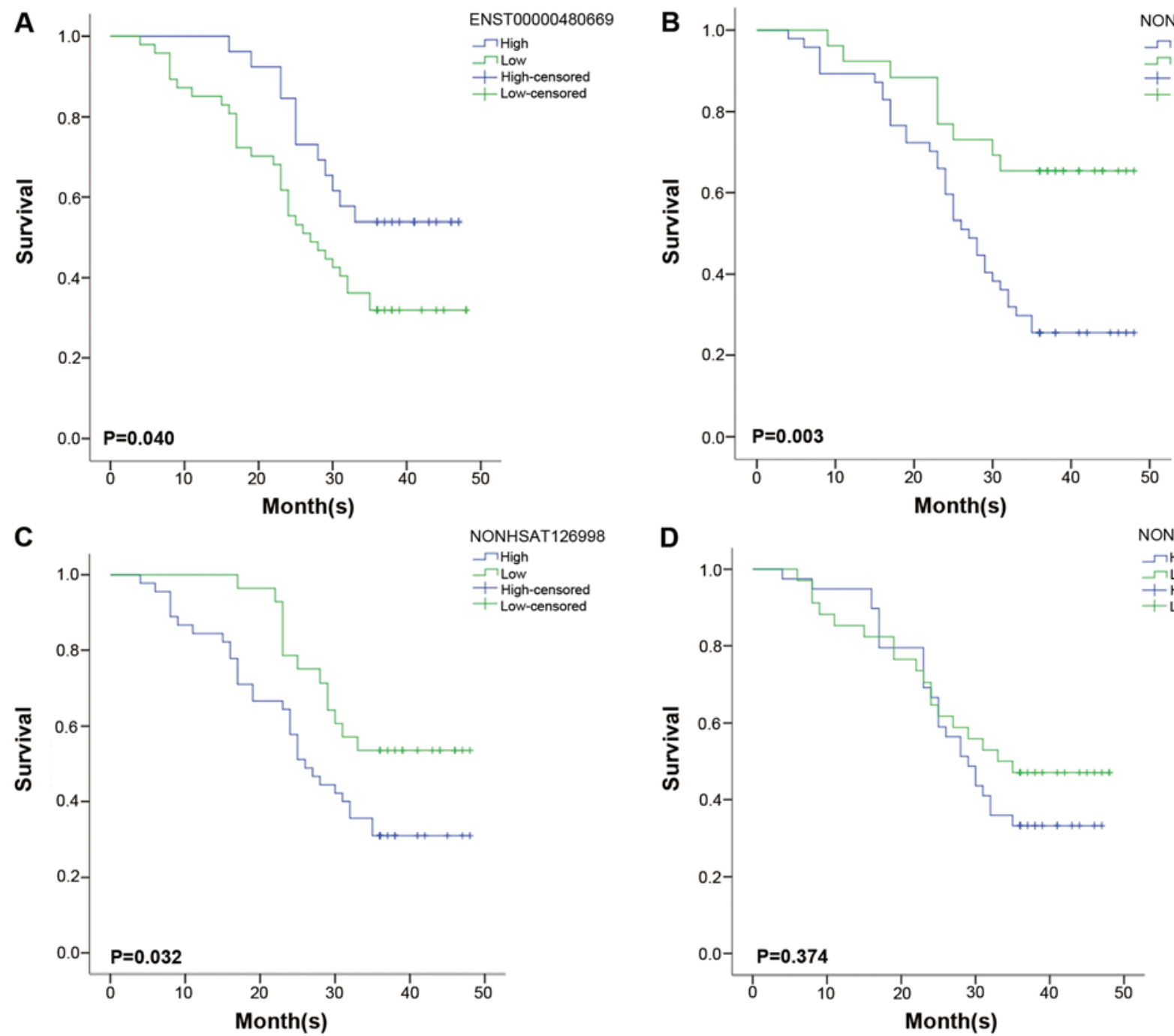

Figure 8. The 3-year survival rate of the selected IncRNAs. (A) The 3-year survival curve of ENST00000480669. (B) The 3-year survival curve of NONHSAT104436. (C) The 3-year survival curve of NONHSAT126998. (D) The 3-year survival curve of NONHSAT112918.

Table IX. Multivariate Cox regression analysis for ENST00000480669, NONHSAT104436, NONHSAT126998 and NONHSAT112918.

\begin{tabular}{lccccccr}
\hline & & & & & & \multicolumn{2}{c}{$95 \%$ CI for Exp(B) } \\
\cline { 5 - 8 } & B & SE & Wald & Sig. & Exp(B) & Lower & Upper \\
\hline ENST00000480669 & -0.597 & 0.377 & 2.513 & 0.113 & 0.550 & 0.263 & 1.152 \\
NONHSAT104436 & 1.149 & 0.482 & 5.675 & 0.017 & 3.156 & 1.226 & 8.123 \\
NONHSAT12699 & -0.279 & 0.460 & 0.367 & 0.545 & 0.757 & 0.773 & 2.598 \\
NONHSAT112918 & 0.349 & 0.309 & 1.273 & 0.259 & 1.417 & 0.307 & 1.865 \\
\hline
\end{tabular}

(Fig. 8). From the multivariate Cox regression analysis, only NONHSAT104436 was an independent prognostic factor ( $\mathrm{P}=0.017$; 95\% CI, 1.226-8.123; Table IX).

\section{Discussion}

Recently, IncRNAs were reported to be involved in numerous biological process and be connected with various diseases, such as cancer (27-29), and many lncRNAs play critical roles in regulating gene expression $(30,31)$. Since AFAP1-AS1 (an IncRNA transcript) was demonstrated differentially expressed in esophageal adenocarcinoma, an increasing number of aberrant expression of lncRNAs have been reported in esophageal cancer (32). However, the understanding of the genome-wide expression patterns and functions of lncRNAs in ESCC is still limited. 
In this study, we examined the profiles of IncRNA expression in ESCC tissues and matched non-cancerous tissues by microarray assay and identified 182 lncRNAs with statistically significant different expression patterns. Then, we performed an integrated analysis of these lncRNAs, concentrating on IncRNA co-expressed gene analyses, gene ontology and pathway analyses, target gene prediction analyses to explore their potential function and target genes in ESCC. Finally, we selected four dysregulated lncRNAs (ENST00000480669, NONHSAT104436, NONHSAT126998 and NONHSAT112918) to validate their expression patterns in patients with ESCC by qRT-PCR. The four lncRNAs showed significant correlation to certain clinicopathological features, including lymph node metastasis, tumor infiltrating stage, distant metastasis and TNM stage. ENST00000480669, NONHSAT104436 and NONHSAT126998 were related to the prognosis of ESCC in cancer patients. Among the four aberrant lncRNAs, only NONHSAT104436 was an independent prognostic factor.

It is known that the expression of a single lncRNA could be correlated with hundreds of coding genes. Therefore, it is a big challenge to decipher the functions of IncRNAs. Compelling evidence has shown that similar expression patterns of genes potentially shared related functions or were involved in the same biological pathways $(33,34)$. The GO concept used a common vocabulary to query and retrieve gene and gene product based on their core biological functions through a dynamic and flexible way in multiple organisms (35). Here, we constructed coexpression of coding-non-coding genes and used GO and pathway analysis to predict the lncRNA functions in ESCC. Based on our data, the main enriched biological processes in predicting differently expressed lncRNAs were closely tied up to ESCC development and progression, such as 'extracellular matrix', 'immune responses', 'cell differentiation', 'cell proliferation'. In the above main enriched terms from GO analysis, the most significant GO term in biological processes was 'extracellular matrix', indicating that dysregulated lncRNAs could play the leading role in regulating extracellular matrix expression. While the extracellular matrix is the first barrier to hold back the metastasis of tumor, based on the significant KEGG pathways analyses, the most correlated pathways were 'cell cycle', 'ECM-receptor interaction', 'pathways in cancer', 'TGF- $\beta$ signaling pathway' and 'transcriptional misregulation in cancer', which also proved that the aberrant lncRNAs may play an crucial role in ESCC development and progression. In these IncRNAs, NONHSAT104436 drew our attention, as it was the most upregulated in the 182 significantly differently expressed IncRNAs, and it was significantly associated with SOX2 (PCC, -0.99), whose function has been confirmed in ESCC and was consistent with GO and pathway analysis.

Because of the diverse and complex functions of the lncRNAs, the molecular regulatory mechanisms of lncRNAs remain unknown. The function of IncRNAs has been reported to regulate their own transcriptions or their neighboring coding genes by cis-regulatory mechanisms $(36,37)$, which was regarded as an lncRNA intrinsic capacity (38). The cis-regulatory mechanism was reported to be used as one of the methods to predict the IncRNA target genes (23). In this study, there are 50 significantly different expressed lncRNAs that regulate
85 mRNAs by cis regulatory mechanisms. With regard to their expression changes, 74 pairs demonstrated positive correlation, and 11 pairs have negative correlation. Among these genes, VEGFA was the cis gene of IncRNA NONHSAT112918, and its biological function has been confirmed in ESCC by a large number of scientific studies. As the target genes were regulated by each corresponding IncRNA, expression change of lncRNAs in ESCC tissue may influence the expression of the target genes, and these IncRNAs may affect ESCC development and progression. Through analyzing the cis, we may gain the target genes of lncRNAs and more information about their regulatory mechanisms in ESCC.

However, the lncRNA co-expressed encoding genes mostly lie in different regions of the same chromosome, or even in different chromosomes. So it is not enough to predict the target gene of lncRNAs by using the cis regulation mechanism only. The trans regulation mechanism of IncRNAs can regulate the expression of target genes on a different locus. It was reported that many lncRNAs interact with transcription factors, and increasing evidence proves the trans mechanism in lncRNAs. Jiang et al and Yang et al developed web-based tools to provide integrated views for common transcription factors and lncRNA genes based on ChIP-Seq data $(39,40)$. Lopez-Pajares et al constructed an IncRNA-TF network for epidermal differentiation (41). Based on trans-regulatory mechanism, this study demonstrated 168 lncRNAs were regulated by 95 TFs. Through cluster analysis, we found most of the aberrant IncRNAs co-expressed mRNAs enriched in $20 \mathrm{TFs}$, which may play an important role in regulating lncRNA expression in ESCC. Three TFs E2F1, BCLAF1 and SP1 of the above 20 TFs have been reported in ESCC (24-26). The biological meaning of the elevated expression of TFs GTF2F1, SIN3A and IRF3 in ESCC remain to be validated. We explored the relationship of lncRNA-TFs and lncRNA-TFs-target genes. The results showed lncRNAs ENST00000480669 and NONHSAT126998 were significantly associated with E2F1 and BCLAF1, which indicated that they may have important function in ESCC.

Aberrant lncRNAs have been reported to be involved in tumorigenesis, invasion and metastasis (15-17). Investigating differential expression of IncRNAs in various tumor tissues may provide new understanding for cancer diagnosis, prognosis and targeted therapy. For instance, the IncRNA HOTAIR expression was elevated in primary and metastasizing breast tumors, and the expression level of HOTAIR in primary tumors was a strong predictor of final metastasis and prognosis (29). Li et al suggested that a three-lncRNA signature containing lncRNAs XLOC_013014, ENST00000435885.1 and ENST00000547963.1 was a novel biomarker for the prognosis of ESCC (42). High-throughput cancer genome sequencing also have identified valuable biomarkers in ESCC $(4,43)$. In this study, we first reported four lncRNAs whose coding genes had been proved positively correlated with ESCC and the relationship among those four lncRNAs and clinical clinicopathological features, prognosis were analyzed in 73 patients with ESCC. We discovered all the four were significantly related with one or more clinicopathological features. More importantly, the ESCC patients with high expression of NONHSAT104436 were vulnerable to cancer metastasis. Further univariate survival analysis 
demonstrated that ESCC patients with low expression of ENST00000480669 had a markedly decreased survival rate in a 3-year survey. In addition, ESCC patients with low expression of NONHSAT126998 or NONHSAT104436 had a better prognosis. These results indicate that ENST00000480669, NONHSAT104436 and NONHSAT126998 were worth exploring in predicting prognosis for ESCC patients. Significantly, in the multivariate analysis, the retrospective study of 73 ESCC patients indicated that NONHSAT104436 was the only independent prognostic factor in ESCC. This result provides that NONHSAT104436 may be a promising biomarker for diagnosis and prognosis of ESCC.

\section{Acknowledgements}

This study was supported by the Health Foundation (grant no. H201260) of Jiangsu Province, China, the Social Development Foundation (Grant no. TS029) of Taizhou municipal government, China. The authors are grateful to Dr Xueliang Han, for his assistance with the manuscript preparation.

\section{References}

1. Jemal A, Bray F, Center MM, Ferlay J, Ward E and Forman D: Global cancer statistics. CA Cancer J Clin 61: 69-90, 2011.

2. Chen W, Zheng R, Zeng H, Zhang S and He J: Annual report on status of cancer in China, 2011. Chin J Cancer Res 27: 2-12, 2015

3. Gao Y, Hu N, Han X, Giffen C, Ding T, Goldstein A and Taylor P: Family history of cancer and risk for esophageal and gastric cancer in Shanxi, China. BMC Cancer 9: 269, 2009.

4. Song Y, Li L, Ou Y, Gao Z, Li E, Li X, Zhang W, Wang J, Xu L, Zhou Y, et al: Identification of genomic alterations in oesophageal squamous cell cancer. Nature 509: 91-95, 2014.

5. Bartel DP: MicroRNAs: Genomics, biogenesis, mechanism, and function. Cell 116: 281-297, 2004

6. Lewis BP, Burge CB and Bartel DP: Conserved seed pairing, often flanked by adenosines, indicates that thousands of human genes are microRNA targets. Cell 120: 15-20, 2005.

7. Png KJ, Halberg N, Yoshida M and Tavazoie SF: A microRNA regulon that mediates endothelial recruitment and metastasis by cancer cells. Nature 481: 190-194, 2011.

8. Isozaki Y, Hoshino I, Akutsu Y, Hanari N, Mori M, Nishimori T, Murakami K, Akanuma N, Takeshita N, Maruyama T, et al: Usefulness of microRNA-375 as a prognostic and therapeutic tool in esophageal squamous cell carcinoma. Int J Oncol 46 1059-1066, 2015.

9. Spizzo R, A1meida MI, Colombatti A and Calin GA: Long non-coding RNAs and cancer: A new frontier of translational research? Oncogene 3 1: 4577-4587, 2012.

10. Necsulea A, Soumillon M, Warnefors M, Liechti A, Daish T, Zeller U, Baker JC, Grützner F and Kaessmann H: The evolution of lncRNA repertoires and expression patterns in tetrapods. Nature 505: 635-640, 2014.

11. Yang L, Duff MO, Graveley BR, Carmichael GG and Chen LL: Genome-wide characterization of non-polyadenylated RNAs. Genome Biol 12: R16, 2011.

12. Caley DP, Pink RC, Trujillano D and Carter DR: Long noncoding RNAs, chromatin, and development. Sci World J 10 90-102, 2010.

13. Mercer TR, Dinger ME and Mattick JS: Long non-coding RNAs: Insights into functions. Nat Rev Genet 10: 155-159, 2009.

14. Guttman M, Amit I, Garber M, French C, Lin MF, Feldser D, Huarte M, Zuk O, Carey BW, Cassady JP, et al: Chromatin signature reveals over a thousand highly conserved large noncoding RNAs in mammals. Nature 458: 223-227, 2009.

15. Yuan JH, Yang F, Wang F, Ma JZ, Guo YJ, Tao QF, Liu F, Pan W, Wang TT, Zhou CC, et al: A long non-coding RNA activated by TGF- $\beta$ promotes the invasion-metastasis cascade in hepatocellular carcinoma. Cancer Cell 25: 666-681, 2014
16. Gupta RA, Shah N, Wang KC, Kim J, Horlings HM, Wong DJ, Tsai MC, Hung T, Argani P, Rinn JL, et al: Long non-coding RNA HOTAIR reprograms chromatin state to promote cancer metastasis. Nature 464: 1071-1076, 2010.

17. Qiu JJ, Lin YY, Ding JX, Feng WW, Jin HY and Hua KQ: Long non-coding RNA ANRIL predicts poor prognosis and promotes invasion/metastasis in serous ovarian cancer. Int J Oncol 46: 2497-2505, 2015

18. Yang H, Liu Z, Yuan C, Zhao Y, Wang L, Hu J, Xie D, Wang L and Chen D: Elevated JMJD1A is a novel predictor for prognosis and a potential therapeutic target for gastric cancer. Int J Clin Exp Pathol 8: 11092-11099, 2015.

19. Kim K, Jutooru I, Chadalapaka G, Johnson G, Frank J, Burghardt R, Kim S and Safe S: HOTAIR is a negative prognostic factor and exhibits pro-oncogenic activity in pancreatic cancer. Oncogene 32: 1616-1625, 2013.

20. Martínez-Fernández M, Rubio C, Segovia C, López-Calderón FF, Dueñas $\mathrm{M}$ and Paramio JM: EZH2 in Bladder Cancer, a Promising Therapeutic Target. Int J Mol Sci 16: 27107-27132, 2015.

21. Gerstein MB, Kundaje A, Hariharan M, Landt SG, Yan KK, Cheng C, Mu XJ, Khurana E, Rozowsky J, Alexander R, et al: Architecture of the human regulatory network derived from ENCODE data. Nature 489: 91-100, 2012.

22. Guttman M and Rinn JL: Modular regulatory principles of large non-coding RNAs. Nature 482: 339-346, 2012.

23. Wang J, Xie H, Ling Q, et al: Coding-non-coding gene expression in intrahepatic cholangiocarcinoma. Transl Res 168: 107-121, 2016.

24. Lu XF, Li EM, Du ZP, Xie JJ, Guo ZY, Gao SY, Liao LD, Shen ZY, $\mathrm{Xie} D$ and Xu LY: Specificity protein 1 regulates fascin expression in esophageal squamous cell carcinoma as the result of the epidermal growth factor/extracellular signal-regulated kinase signaling pathway activation. Cell Mol Life Sci 67: 3313-3329, 2010.

25. Yeo SY, Ha SY, Yu EJ, Lee KW, Kim JH and Kim SH: ZNF282 (Zinc finger protein 282), a novel E2F1 co-activator, promotes esophageal squamous cell carcinoma. Oncotarget 5: 12260-12272, 2014.

26. Chen Y, Wang Y, Song H, Wang J, Yang H, Xia Y, Xue J, Li S, Chen $\mathrm{M}$ and $\mathrm{Lu} \mathrm{Y}$ : Expression profile of apoptosis-related genes potentially explains early recurrence after definitive chemoradiation in esophageal squamous cell carcinoma. Tumour Biol 35: 4339-4346, 2014

27. Rinn JL and Chang HY: Genome regulation by long non-coding RNAs. Annu Rev Biochem 81: 145-166, 2012.

28. Chen G, Wang Z, Wang D, Qiu C, Liu M, Chen X, Zhang Q, Yan $G$ and Cui Q: LncRNADisease: A database for long-noncoding RNA-associated diseases. Nucleic Acids Res 41D: D983-D986, 2013.

29. Gutschner T and Diederichs S: The hallmarks of cancer: A long non-coding RNA point of view. RNA Biol 9: 703-719, 2012.

30. Huarte M: LncRNAs have a say in protein translation. Cell Res 23: 449-451, 2013.

31. Nagano T and Fraser P: No-nonsense functions for long noncoding RNAs. Cell 145: 178-181, 2011.

32. Hao Y, Wu W, Shi F, Dalmolin RJ, Yan M, Tian F, Chen X, Chen G and Cao W: Prediction of long non-coding RNA functions with co-expression network in esophageal squamous cell carcinoma. BMC Cancer 15: 168, 2015.

33. Lee HK, Hsu AK, Sajdak J, Qin J and Pavlidis P: Coexpression analysis of human genes across many microarray data sets. Genome Res 14: 1085-1094, 2004

34. Eisen MB, Spellman PT, Brown PO and Botstein D: Cluster analysis and display of genome-wide expression patterns. Proc Natl Acad Sci USA 95: 14863-14868, 1998.

35. Ashburner M, Ball CA, Blake JA, Botstein D, Butler H, Cherry JM, Davis AP, Dolinski K, Dwight SS, Eppig JT, et al; The Gene Ontology Consortium: Gene ontology: Tool for the unification of biology. Nat Genet 25: 25-29, 2000.

36. Wang KC, Yang YW, Liu B, Sanyal A, Corces-Zimmerman R, Chen Y, Lajoie BR, Protacio A, Flynn RA, Gupta RA, et al: A long non-coding RNA maintains active chromatin to coordinate homeotic gene expression. Nature 472: 120-124, 2011.

37. Guenzl PM and Barlow DP: Macro lncRNAs: A new layer of cis-regulatory information in the mammalian genome. RNA Biol 9: 731-741, 2012. 
38. Lee JT: Lessons from X-chromosome inactivation: Long ncRNA as guides and tethers to the epigenome. Genes Dev 23: 1831-1842, 2009.

39. Jiang Q, Wang J, Wang Y, Ma R, Wu X and Li Y: TF2LncRNA: Identifying common transcription factors for a list of lncRNA genes from ChIP-Seq data. BioMed Res Int 2014: 317642, 2014.

40. Yang JH, Li JH, Jiang S, Zhou H and Qu LH: ChIPBase: A database for decoding the transcriptional regulation of long non-coding RNA and microRNA genes from ChIP-Seq data. Nucleic Acids Res 41 (D1): D177-D187, 2013.

41. Lopez-Pajares V, Qu K, Zhang J, Webster DE, Barajas BC, Siprashvili Z, Zarnegar BJ, Boxer LD, Rios EJ, Tao S, et al: A LncRNA-MAF:MAFB transcription factor network regulates epidermal differentiation. Dev Cell 32: 693-706, 2015.
42. Li J, Chen Z, Tian L, Zhou C, He MY, Gao Y, Wang S, Zhou F, Shi S, Feng X, et al: LncRNA profile study reveals a threelncRNA signature associated with the survival of patients with oesophageal squamous cell carcinoma. Gut 63: 1700-1710, 2014.

43. Dulak AM, Stojanov P, Peng S, Lawrence MS, Fox C, Stewart C, Bandla S, Imamura Y, Schumacher SE, Shefler E, et al: Exome and whole-genome sequencing of esophageal adenocarcinoma identifies recurrent driver events and mutational complexity. Nat Genet 45: 478-486, 2013. 